\title{
Improving Efficiency and Equity in Early Autism Evaluations: The (S)TAAR Model
}

\author{
Meredith I. Brinster ${ }^{1}$ - Briana H. Brukilacchio ${ }^{2}$. Autumn Fikki-Urbanovsky ${ }^{2}$ Jeffrey D. Shahidullah ${ }^{1}$ (D) \\ Sheri Ravenscroft ${ }^{3}$
}

Accepted: 2 January 2022 / Published online: 12 January 2022

(c) The Author(s), under exclusive licence to Springer Science+Business Media, LLC, part of Springer Nature 2022

\begin{abstract}
This paper describes the (Sin Exclusión) Transdisciplinary Autism Assessment and Resources ([S]TAAR) model and presents early metrics tracking efficiency and equity in access to high-quality comprehensive autism evaluations for young children. Retrospective chart reviews over one year (08/2018-08/2019) with $n=173$ children were reviewed. Through care coordination with community providers, the model was developed to meet local needs by increasing throughput of children ( $<4$ years old) evaluated by a transdisciplinary team. Team-based processes included pre-visit triage, synchronous evaluation procedures, case conferencing, huddles, and care coordination. The model led to increased patient throughput, reductions in waitlist and time to diagnosis, and improved provider satisfaction. Improvements in access to care were equitable across patient race, ethnicity, language, and insurance type.
\end{abstract}

Keywords Autism $\cdot$ Autism spectrum disorder $\cdot$ Evaluation $\cdot$ Interprofessional $\cdot$ Team-based

\section{Introduction}

Autism spectrum disorder (hereafter, autism) is among the most commonly diagnosed neurodevelopmental disabilities (currently 1 in 44 children). Early identification is critical for connecting children and families to intervention services

Jeffrey D. Shahidullah

jeff.shahidullah@austin.utexas.edu

Meredith I. Brinster

meredith.brinster@ascension.org

Briana H. Brukilacchio

brianahb@utexas.edu

Autumn Fikki-Urbanovsky

autumnfikki@utexas.edu

Sheri Ravenscroft

sravenscroft@ascension.org

1 Department of Psychiatry and Behavioral Sciences, Dell Medical School, The University of Texas at Austin, Health Discovery Building, 1601 Trinity St., Bldg B, Z0600, Austin, TX 78712, USA

2 Department of Educational Psychology, The University of Texas at Austin, Austin, TX, USA

3 Division of Pediatrics, The University of Texas at Austin, Austin, TX, USA to promote positive developmental, behavioral, social-emotional, and educational outcomes. Still, significant delays persist between caregivers' reported concerns to healthcare professionals, subsequent referrals for specialty evaluation, and eventual identification (Zwaigenbaum et al., 2015; Bishop-Fitzpatrick et al., 2017; Zablotsky et al., 2017). Although children with autism often present with developmental concerns by 12 months of age, the majority are identified after age three, when eligibility for state-funded early intervention services typically ends. One-third to onehalf of children are identified after school age (Baio et al., 2018; Sheldrick et al., 2017). Minoritized children and children of low socio-economic status are typically diagnosed even later (Durkin et al., 2010). Families with limited English proficiency also have increased difficulty in accessing developmental specialists, such as developmental behavioral pediatricians, who provide rigorous diagnostic evaluations (Jimenez et al., 2017). Families' experience of this lengthy and arduous process has been termed a "diagnostic odyssey" (Lappe et al., 2018).

This odyssey for families typically begins in primary care and/or early childhood special education programs. In the primary care context, the American Academy of Pediatrics has disseminated screening guidelines for well-child visits to identify patients who may benefit from comprehensive 
autism testing (Lipkin et al., 2020; Dreyer et al., 2016). However, there is a lack of consistent and standardized use of screening and referral processes within primary care, which hampers early autism diagnosis (Radecki et al., 2011; Zuckerman et al., 2015). In the school context, federallyfunded early childhood special education programs also evaluate and provide services to children under age four with developmental delays or behavioral symptoms. School districts are responsible for identifying children in their local communities and providing timely educational evaluations and treatment planning. However, in some states, such as BLINDED, children with only an educational disability are not eligible for intensive interventions provided through the healthcare system, including applied behavior analysis (ABA). Each of these settings collects valuable pieces of information that can potentially be useful for timely diagnostic decision-making. Unfortunately, information sharing across providers and settings can be hampered by privacy laws (e.g., FERPA, HIPAA) and lack of purposeful care coordination (Shahidullah et al., 2018, 2020).

Poor care coordination across early childhood settings contributes to inefficiencies, delays, and fragmentation with care-seeking as well as marginalization and emotional stressors for parents seeking services on behalf of their child. Parents of children with autism report feeling overwhelmed, unheard, and isolated throughout the diagnostic process (Lappe et al., 2018; Woodgate et al., 2008). Minoritized families (i.e., Black, Latinx) experience additional marginalization as they report racial bias in interactions with healthcare professionals or culturally-specific stigma and fears related to developmental testing and disability (Dababnah et al., 2018).

Nationally, a number of innovative models have targeted inefficiencies in the diagnostic odyssey to improve family experience and satisfaction with care. Streamlined referral pathways (Hine et al., 2018), family navigation (Crossman et al., 2020), multidisciplinary team approaches (GordonLipkin et al., 2016; Gerdts et al., 2018; Williams-Arya et al., 2019; Koushnik et al., 2015), and improvement projects using systems analysis (Austin et al., 2016) have demonstrated promise in improving access and efficiency in early childhood autism evaluations. Key takeaways include the ongoing need to redesign clinical services for early autism identification, with an emphasis on functional improvements over diagnostic labeling, sensitivity to the caregiver's experience (Gordon-Lipkin et al., 2016), improved care coordination (Parker et al., 2019), and a focus on social equity (Phelps \& Coker, 2019).

\section{Context and Goals}

In Texas, there are a number of unique barriers in equitable access to early autism evaluations. Texas is a "majority-minority" state with equal populations of Hispanic and Non-Hispanic White residents and over 35\% of Texans speak a language other than English. Over 3.5 million children in Texas rely on Medicaid and CHIP to access healthcare (Georgetown University Health Policy Institute, Center for Children and Families, 2021). More than half of families of children with autism in the state report experiencing problems with obtaining referrals, $47 \%$ report having unmet care coordination needs, and $24 \%$ report experiencing difficulty utilizing services (Thomas et al., 2012).

In the Central Texas catchment area there are very few providers specializing in autism assessment who accept insurance, including Medicaid and managed Medicaid plans. Many families from rural areas travel considerable distances to attend visits with these specialists and have experienced long appointment waitlists. Deriving from previously reported models and clinical innovations, we developed an autism diagnostic model to address the barriers to care in this region with an explicit commitment to equity and cultural sensitivity. The (Sin Exclusión) Transdisciplinary Autism Assessment and Resources ([S]TAAR) model integrates systems-level and family-centered approaches to improve access to early autism evaluations without exacerbating existing healthcare inequities. This study describes patient flow and program effectiveness throughout its initial implementation, with a focus on care coordination and social equity.

\section{Setting Description}

The (S)TAAR model was developed through a collaboration between the Division of Developmental Behavioral Pediatrics at Dell Children's Medical Center and the Department of Psychiatry and Behavioral Sciences within Dell Medical School at The University of Texas at Austin. Program development was led by a developmental behavioral pediatrician (DBP) and pediatric psychologist, each with specialized training in early autism assessment. Prior to implementing the (S)TAAR model, new patients referred to the Division of Developmental Behavioral Pediatrics at Dell Children's Medical Center for suspected autism were scheduled for multiple visits including intake, testing, feedback, and follow-up with the DBP. All patients generally followed the same referral stream, regardless of age and reason for referral. At the time, waitlists for new patients exceeded well over 1 year.

\section{Referral Sources}

The (S)TAAR model was developed for new patients with concern for autism who were under the age of four years at the time of referral. Referral sources included primary care clinicians, high-risk neonatal follow-up programs, and 
early childhood intervention programs. When case managers and early interventionists suspected autism, they obtained permission from the family to send a templated letter to the primary care clinician outlining their concerns and requesting that a referral be made to the (S)TAAR clinic to initiate the evaluation process. Many of the referred patients were already participating in federally-funded early childhood intervention (ECI) programs, which often allowed for case managers and interventionists to flag behaviors concerning for autism. Additionally, ECI programs, and a subset of the medical referral sources, regularly use standardized developmental assessment measures (e.g., Battelle Developmental Inventory, Second Edition [BDI-2; Newborg, 2009]; Bayley Scales of Infant and Toddler Development, Third Edition [Bayley-III; Bayley, 2005]; Developmental Assessment of Young Children, Second Edition [DAYC-2; Voress et al., 2012]) to qualify for services and for progress monitoring. As part of the triage process, the (S)TAAR clinic nurse coordinator contacted parents and providers to obtain copies of these evaluations as well as other medical and educational records prior to the appointment (via clinic email or fax). Available records were reviewed and used in case conceptualization and planning for appointment procedures. Recent developmental testing (i.e., within three months of appointment) was often not fully repeated at the clinic appointment.

\section{Identification-Initial Triage}

The clinic nurse coordinator reviewed physician referrals and developmental concerns listed in the clinical intake paperwork to identify patients meeting inclusion criteria for the (S)TAAR program: patients under the age of 4 with a chief complaint of developmental delay, behavior, speech, or autism. Referrals may include developmental screeners from well-child visits (e.g., Modified Checklist for Autism in Toddlers [M-CHAT-R/F]; Robins et al., 2009) or evaluations from local school districts or community programs (e.g., Battelle Developmental Inventory, Second Edition). A minority of patients in the (S)TAAR program may also present with provisional diagnoses of autism seeking second opinions or more comprehensive evaluations.

As part of this pre-visit triage, (S)TAAR patients are categorized into one of two referral groups: patients with available behavioral screening or developmental testing are assigned to the DATA group; patients without available previous developmental or screening data are assigned to the NADA group. In addition to assisting in the pre-visit case conference, the primary purposes of this categorization process are to: (1) provide structure and balance to the demands and workflow within the clinic, (2) allow sufficient time for documentation, and (3) tentatively plan specific duties each member of the transdisciplinary team will complete during each patient's visit. NADA appointments typically require less extensive record review and more comprehensive direct assessment in the clinic. DATA appointments typically include more extensive record review and a comparatively brief test battery administered in the clinic.

\section{Team Huddles}

Team huddles include, at minimum, the medical team (developmental behavioral pediatrician, nurse practitioner), psychology team (pediatric psychologist, psychometrist), nurse coordinator, and social work team. Data obtained through medical and educational records are reviewed sequentially during team huddles, including relevant family updates. The team summarizes information from the new patient intake questionnaire (e.g., social needs screening questions, previous therapies and evaluations, DSM-5 screening items) and previous screening or assessment measures (e.g., Modified Checklist for Autism in Toddlers-Revised [M-CHATR/F]; Robins et al., 2009; Ages and Stages Questionnaire [ASQ; Squires \& Bricker, 2019]). Providers outline the developmental and diagnostic tools they planned to use for each patient, begin charting previous evaluation data, and discuss possible referrals. For instance, the social worker would begin to identify which ABA providers serve a family's geographic area and accept their insurance in case an autism diagnosis is provided at the appointment. The team also identifies families who endorsed stressors or structural barriers in their paperwork (e.g., housing or food insecurity) and plans are developed for social work to meet with parents during their child's visit.

\section{Transdisciplinary Assessment}

The (S)TAAR clinical assessment team combines expertise from multiple disciplines and training backgrounds including developmental behavioral pediatrics, pediatric psychology, and social work. Providers have both overlapping and distinct areas of expertise related to early development and evidence-based interventions. Within the (S)TAAR model, the developmental behavioral pediatrician leads the broader team of providers in the evaluation and conceptualization of each case from start to finish. This transdisciplinary (Vyt, 2008) model was intended to minimize redundancy from the perspective of the family, increase cohesion across specialties, and ensure that each patient is considered and supported with relation to their medical symptoms, social determinants of health, and psychosocial factors. The assessment procedures, intervention recommendations, and post-diagnostic family support are individualized and tightly aligned with the value-based model of healthcare by providing maximal expertise through coordinated and team-based care at minimal cost to patients in terms of visit duration, number of appointments, and billing structure. 
Practically, the visit consists of physical examination, a synchronous clinical/developmental interview, administration of developmental measures of verbal and nonverbal reasoning as needed, and completion of semi-structured play-based behavioral observations (e.g., Childhood Autism Rating Scale, 2nd ed; Schopler et al., 2010). Concurrent interviewing and observation allow for efficient collection of data and have proven to engage the child in a way that allows parents to focus on answering questions. This model has successfully accommodated in-person interpreters for families who prefer to receive their care in languages other than English.

\section{Diagnostic Conference}

Towards the conclusion of the 90 min (S)TAAR appointment, providers excuse themselves from the exam room and hold a brief case conference to review clinical impressions, come to a diagnostic consensus, and develop individualized recommendations. Same-day feedback is always provided, although in a small minority of cases that includes a recommendation to return for an additional $60 \mathrm{~min}$ diagnostic appointment before an autism diagnosis may confidently be ruled in/out. Diagnostically, the case conference is intended to safeguard against potential forms of clinician bias that could impact behavioral diagnostics combining multiple clinical perspectives to address the many variables impacting early development and behavioral dysregulation. During this brief discussion, those who primarily took behavioral observations and those who primarily focused on interviewing are able to better understand the various sources of data and quickly consider these in combination.

During the case conference, the team also begins to transition from a primarily diagnostic focus to an intervention focus. In this way, the functional impact of the evaluation is prioritized over the mere provision of a diagnostic label. In service of this aim, the team agrees upon an integrated set of medical, behavioral, and psychosocial recommendations for each patient which align with the families' identified needs and preferences. The primary goal of these recommendations is to quickly connect families with relevant subspecialty care (as needed) as well as early intervention, special education, parent/family support, and community resources. Recommendations are intentionally targeted and numerically ordered in order to minimize the post-diagnostic stress and demands placed upon families.

\section{Feedback}

Feedback with the family is led by the developmental behavioral pediatrician, who emphasizes the child's strengths in the context of referral concerns and diagnostic impressions. Feedback includes transparent discussion of diagnostic differentials as well as explicit examples of critical behavioral observations and caregiver input that contributed to diagnostic decision-making. Additional discussions regarding referrals to appropriate medical follow up (e.g., genetic testing, brain imaging, electroencephalogram, audiology) are also discussed at this time. A major emphasis at the feedback part of the visit is connecting families to appropriate community-based interventions and supports, as well as ongoing support through a warm handoff to the team social worker who further discusses options for intervention, community support, and clinic-based parent education and social support programs.

\section{Follow-up}

If further assessment procedures are recommended, the family is referred to the team's pediatric psychologist. If all diagnostic impressions and feedback are completed during the initial appointment, families are scheduled for a followup "bridge" appointment with the team social worker one month after their diagnosis to assist in securing the recommended supports and interventions. Finally, families are scheduled for a 4- to 6-month follow-up appointment with one of the team's medical providers (FNP-C, MD) to further ensure access to subspecialty services, evaluate functional progress, and discuss results from any medical referrals (e.g., genetic testing, neuroimaging, audiology). See Holley et al. (2020) for additional detail on the visit procedures associated with (S)TAAR.

\section{Methods}

Study approval was obtained by the University of Texas at Austin IRB and covered entity. Analyses are descriptive using chart reviews and related to efficiency and equity metrics corresponding to the first year that (S)TAAR was implemented in the clinic setting. Data collection spanned the initial 12 months of implementation and was managed using Research Electronic Data Capture software (REDCap; Harris et al., 2009). Retrospective data was compiled from the 173 patients who completed an evaluation in the STAAR clinic from August 2018 to August 2019. Patients were not excluded from this diagnostic model based on sex, primary language, insurance type, or clinical complexity.

To guard against bias, research assistants with no connection to the clinical model reviewed medical records to identify relevant variables, including patient demographics, timelines associated with the diagnostic process, method of payment/insurance status, visit procedures, diagnoses, and recommendations. Prior to entering patient data into REDCap, five research assistants were trained to $90 \%$ inter-rater agreement using practice records and a two-step verification 
process was utilized during chart abstraction. Twenty percent of the overall records were identified at random to estimate percent agreement across raters, which averaged $96.23 \%$, ranging from $90.9 \%$ to $99.58 \%$. Given this study's focus on efficiency and equity, efficiency was represented as the average time-to-diagnosis, overall patient throughput, and degree of care coordination (e.g., results from diagnostic screening instruments administered in primary care). Equity was evaluated by testing the extent to which these outcomes varied according to patients' race, ethnicity, primary language, and insurance type.

A brief provider satisfaction survey was developed and disseminated via REDCap to clinicians, support staff, and student trainees who had participated in (S)TAAR visits. Questions were based on a similar survey described by Gerdts et al. (2018). Respondents were asked to rate on a scale from 1 to 5 their happiness during the workday; confidence with diagnostic decision-making; confidence providing feedback to families; workload manageability; and the extent to which they developed professional skills through the clinic visits. Respondents were also prompted to share feedback in an open response format.

\section{Results}

\section{Patient Characteristics}

Demographic information for the $(n=173)$ patients evaluated within the (S)TAAR model is described in Table 1. Racial identity was diverse (49.7\% White, $11 \%$ Black/African American, 7.5\% Asian, 2.9\% American Indian or Alaska Native, and $12.1 \%$ other). Nearly half ( $45.1 \%)$ of patients identified their ethnicity as Hispanic or Latino and $15.6 \%$ of families listed Spanish as their preferred language. The sample was $72.3 \%$ male, reflecting sex differences associated with autism rates. Half (50.3\%) of patients received some form of public insurance (e.g., Medicaid). The average age of patients at time of referral was 2 years 5 months.

\section{Efficiency}

By using the combined expertise of a transdisciplinary team, clinic efficiency improved while continuing to serve a highrisk population seeking ASD evaluation. Over 12 months of implementation the wait time for the targeted age range was reduced by $77 \%$ (average of 18 months to average of 4 months). Moreover, the broader clinical throughput experienced a $190 \%$ increase in the number of new patients seen per month (average of 19 per month to average of 55 per month). The average time-to-diagnosis for (S)TAAR patients, which spans the period between referral and feedback, was 2.4 months. Patients with a higher degree of
Table 1 Patient characteristics, $n=173$

\begin{tabular}{ll}
\hline Age in months at referral, mean (SD) & $29.15(7.67)$ \\
Sex, $n(\%)$ & $125(72.3)$ \\
Male & $48(27.7)$ \\
Female & \\
Race, $n(\%)$ & $86(49.7)$ \\
White & $19(11)$ \\
Black/African American & $13(7.5)$ \\
Asian & $5(2.9)$ \\
American Indian or Alaska Native & $21(12.1)$ \\
Other & $29(16.8)$ \\
Declined to answer & \\
Ethnicity, $n(\%)$ & $78(45.1)$ \\
Hispanic or Latino & $76(43.9)$ \\
Not Hispanic or Latino & $19(11)$ \\
Declined to answer & \\
Language, $n(\%)$ & $141(81.5)$ \\
English & $27(15.6)$ \\
Spanish & $3(1.7)$ \\
Other & \\
Insurance type, $n(\%)$ & $87(50.3)$ \\
Public & $78(45.1)$ \\
Private & $6(3.5)$ \\
Self-Pay & \\
\hline
\end{tabular}

Percentages not adding up to $100 \%$ is due to missing responses

care coordination during the diagnostic process (e.g., data made available through early childhood special education and/or primary care) obtained a diagnosis more quickly (2.04 months) than those without pre-visit data available for review (2.92 months).

A large majority (95.7\%) of patients who were enrolled in early childhood special education prior to their (S)TAAR appointment had recent standardized developmental assessments or screeners (e.g., ASQ; BDI-2, Bayley-III, DAYC-2) available for review by the (S)TAAR team $(n=45$ of 47$)$. In contrast, only $35.8 \%$ of patients ( $n=39$ of 109$)$ who were not enrolled in early childhood special education had recent, standardized developmental assessments available for team review. Of the patients who had no records available for review prior to their appointment $(n=60), 96.6 \%$ were not enrolled in early childhood special education programs. Fewer than half of all referrals included documentation regarding the use or result of an autism screening instrument (e.g., MCHAT-R/F). See Table 2 for summary.

\section{Equity}

There were no significant differences in time-to-diagnosis based on patient race, ethnicity, language, or insurance type (Table 3). Given that the availability of pre-visit data 
Table 2 Care coordination and availability of pre-visit data

\begin{tabular}{llll}
\hline & Full sample & $\begin{array}{l}\text { Coordination with } \\
\text { primary care only }\end{array}$ & $\begin{array}{l}\text { Coordination with } \\
\text { primary care \& early } \\
\text { intervention }\end{array}$ \\
\hline Clinical data from referral, $n(\%)$ & $(n=175)$ & $(n=109)$ & $(n=47)$ \\
Full & $51(29.1)$ & $24(22)$ & $15(31.9)$ \\
Partial & $64(36.6)$ & $27(24.8)$ & $32(68.1)$ \\
None & $60(34.3)$ & $58(53.2)$ & $1(2.1)$ \\
Developmental screener/assessment, $n(\%)$ & & & \\
Yes & $97(55.4)$ & $39(35.8)$ & $45(95.7)$ \\
No & $78(44.6)$ & $70(64.4)$ & $2(4.2)$ \\
Autism diagnostic screener, $n(\%)$ & & & $14(29.8)$ \\
Yes & $71(40.6)$ & $39(35.8)$ & $33(70.2)$ \\
No & $104(59.4)$ & $70(64.4)$ & \\
\hline
\end{tabular}

Table 3 Diagnostic efficiency according to patient characteristics

\begin{tabular}{ll}
\hline Patient characteristics & Time-to-diagnosis, months (SD) \\
\hline Race & \\
White & $2.26(1.58)$ \\
Black/African American & $2.31(1.86)$ \\
Asian & $2.31(0.95)$ \\
American Indian or Alaska & $2.68(2.28)$ \\
$\quad$ Native & $3.12(2.21)$ \\
Other & $2.57(1.61)$ \\
Declined to answer & $F=0.958, d f=5, p=0.45$ \\
& \\
Ethnicity & $2.49(1.63)$ \\
Hispanic or Latino & $2.44(1.86)$ \\
Not Hispanic or Latino & $2.21(1.14)$ \\
Declined to answer & $F=0.22, d f=2, p=0.80$ \\
& \\
Language & $2.36(1.67)$ \\
English & $2.85(1.85)$ \\
Spanish & $1.87(1.09)$ \\
Other & $3.23(.16)$ \\
Declined to answer & $F=0.891, d f=3, p=0.44$ \\
Insurance type & $2.53(1.57)$ \\
Public & $2.39(1.86)$ \\
Private & \\
& \\
&
\end{tabular}

and increased care coordination were associated with a more efficient diagnostic process, participants were grouped according to the degree of pre-visit data available for review and chi square tests were used to determine whether the patients were equally distributed across these groups. No relationship was identified between the degree of available pre-visit data and patients' ethnicity $(p=0.93)$, race $(p=0.16)$, or preferred language $(p=0.05)$.

\section{Provider Satisfaction}

Seventeen provider satisfaction surveys were collected, representing licensed clinicians $(n=5)$, clinical support staff $(n=3)$, and graduate student trainees $(n=9)$. Disciplines included psychology, social work, developmental behavioral pediatrics, nursing, and speech/hearing sciences. All ratings were on a scale of 1 (low) to 5 (high).

Among clinicians, happiness during the workday was rated an average of 4.8. Confidence with diagnostic decision-making was rated 4.6. Confidence providing feedback to the family was rated 4.8 . And the workload manageability was rated 3.2. Several clinicians $(n=3)$ also conduct evaluations independently within the clinic and provided comparative ratings to reflect their satisfaction in that context. When evaluating young children for autism independently, these clinicians rated their happiness as an average of 3; confidence with the diagnosis was rated 3.33; confidence providing feedback was rated 4 ; and workload manageability was rated 1.33.

Qualitatively, clinicians cited the "shared workload burden," "opportunity to work with other professionals, especially on challenging cases," "having many eyes on the child and input from different disciplines," and "confidence that comes from diagnostic decision making when part of a team" as highlights of the (S)TAAR model. When asked whether they preferred independent versus team evaluations, each clinician with experience in both contexts indicated a preference for the team approach.

When asked about the extent to which (S)TAAR provided professional development opportunities, clinicians and support staff had an average rating of 4.67. Students and trainees had an average rating of 4.73. Qualitatively, students appreciated the model because they were "able to serve so 
many more families," "bounce ideas off of the team to best support the patient's needs," and noted the "convenience for families... like a one-stop shop for them."

\section{Discussion}

\section{Efficiency in Care}

Patients received comprehensive family-centered autism evaluations and were referred to intensive early intervention services within three months of their referral date on average. Like the Arena model (Williams-Arya and et al. (2019), the (S)TAAR model's goal was to reduce wait-times by offering an efficient workflow that maintains the quality of comprehensive autism evaluations through a transdisciplinary approach. This allows clinicians to assume complementary roles and respond to information synchronously to inform clinical decision-making, reduce redundancy, and promote efficiency. This synchronous approach promoted a dynamic and active process whereby evaluation components were streamlined to provide care that was effective from a time and resource perspective.

Efficiency in evaluation services was further enhanced by coordination of care with community early childhood intervention (ECI) providers. This model highlights the availability and utility of previously-collected developmental assessment data, which can be factored into diagnostic decision-making through coordinated care across traditionally siloed service systems. Traditionally, medical and psychological providers administer developmental assessments in-house during the formal evaluation, regardless of the availability of recent community-based developmental testing. In contrast, the (S)TAAR model requests and incorporates recent assessments completed by reliable sources in the community. Advantages of this process in terms of efficiency include eliminating redundant testing, removing barriers to timely diagnosis, and allowing for abbreviated and focused visit procedures.

Using the team huddle strategy, clinician roles were clearly delineated and assigned ahead of time. Clinicians did not need to read through the individual assessment notes from other team members because they were already present as the assessment was taking place. Patient experience was considered by providing families with the opportunity to ask questions of all providers at the team feedback session. These processes led to a reduced burden for the family and healthcare system in terms of billable hours and number of appointments required to obtain a comprehensive diagnostic evaluation. Most families completed this process in the course of one visit, thus reducing travel time and other associated costs (e.g., work leave, child care). From the perspective of the healthcare team, the model also allowed for more families to be seen in less time, thus increasing patient throughput. The informal provider satisfaction survey indicates positive support for the model's ability to increase access for patients and families who are seeking an evaluation. Positive feedback often cited the perceived efficiency of the service (e.g., "we are able to serve so many more families" and described the visits as "like a one-stop shop for [patients and families]." Respondents also reported increased confidence in their diagnostic decision-making and "hope that this confidence is also felt by families, who had received multiple providers' opinions." For respondents who had participated in both (S)TAAR and usual care models (single provider) for this age and referral group, $88 \%$ preferred the (S)TAAR model and $11 \%$ had no preference. Survey respondents also indicated areas for improvement. Several respondents noted the process can feel "overwhelming," or "hectic" due to a lack of physical space to comfortably accommodate the full team and the number of patients seen in one clinic day. Another student shared that the process "felt rushed with some families, especially ones that did not expect to receive an ASD diagnosis or didn't know what ASD is."

Improving workload efficiency through a transdisciplinary model also addresses documented workforce challenges within DBP. These include long waitlists, increasing complexity of DBP patients, excessive workloads, and clerical burdens, which have reportedly driven clinician burnout and moral injury (Bridgemohan et al., 2018; Shanafelt et al., 2012, 2017; West et al., 2018). Given national shortages of DBPs and other developmental specialists, there is a need for models like (S)TAAR that improve patient care while promoting more clinical collaboration through task-shifting (i.e., process of delegation whereby tasks are moved, where appropriate, to less specialized and more available health workers; World Health Organization, 2008). In support of this concept, increased satisfaction with the clinical day emerged as a theme in the provider/staff/trainee survey described above. Specifically, respondents cited the benefits of, "shared workload burden and an opportunity to work with other professionals, especially on challenging cases." Respondents also valued "learning from other providers," "bouncing ideas off one another," and "fast communication with the team as well as multiple perspectives on a single observation of behavior."

This study has also demonstrated support for using previous evaluation data completed by reliable community providers in order to yield more efficient autism evaluations. It is important to highlight the administrative effort involved in requesting and collecting assessment records as a potential barrier for other providers and groups looking to implement similar care coordination. In addition, administration 
support sometimes included care coordination and assistance for families to initiate the referral process directly from primary care clinicians, as required, even when early childhood intervention providers initiate the referral concern.

\section{Equity in Care}

It is crucial that advancements in access to care include equity metrics, given the well-established health disparities in access to autism diagnostic evaluations. As previously recommended by Phelps and Coker (2019), innovative solutions to the autism diagnostic odyssey (sometimes referred to as the "diagnostic bottleneck") must attend to the specific access needs of non-English speaking populations and other medically underserved populations. As emphasized in the (S)TAAR model's name (i.e., Sin Exclusión), the commitment to inclusive and equitable programming was fundamental to program development, implementation, and outcomes monitoring.

Programmatically, by coordinating care with early childhood referral sources and creating a DBP-led transdisciplinary diagnostic team, the (S)TAAR model improved equitable access to autism evaluations in early childhood through a single-visit, transdisciplinary approach. Other similar models of efficient team-based autism evaluations (Seattle Children's Autism Center [SCAC]; Gerdts et al, 2018) have demonstrated similar diagnostic rates and positive provider satisfaction. However, a notable difference in our model's efficiency is what we believe represents a true single-day evaluation for families. Prior to the diagnostic appointment, the SCAC model conducts a separate neurodevelopmental intake and physical examination appointment. Here we propose a model that emphasizes incorporation of existing community-based information (e.g., developmental screening, developmental testing, school-based evaluations) and completes the entire developmental history, physical examination, supplementary developmental testing, and diagnostic decision making in one visit.

Given the large number of Spanish-only speakers in our community and included in this study, it was notable that the use of interpreters did not differentially affect service delivery. In other published models, families of limited English proficiency (LEP) have been excluded from access to team-based evaluations due to the perceived time constraints with the use of interpretation services. Concern that novel approaches to early autism assessment may exacerbate inequities in care has been documented (Phelps \& Coker, 2019). We believe our model is particularly unique, as it demonstrates equitable service delivery across a variety of linguistic and cultural subgroups, including American Sign Language (ASL). With the hope of promoting greater inclusivity in other successful models, these findings are particularly important given that diagnostic bottlenecks differentially impact underserved communities and despite longstanding identification of such disparities for minoritized populations, systemic improvements are scarce.

Future directions may be to evaluate whether telehealth delivery of this model maintains or perhaps increases access to care by reducing other structural barriers (e.g., transportation, driving distances, childcare), particularly in the context of the ongoing Covid-19 pandemic. Procedures are also being piloted to solicit patient and family feedback from a representative sample in terms of language, race, ethnicity, and socioeconomic status. Finally, future iterations of the (S)TAAR model seek to advance the standard of care for Spanish-speaking families by assembling a fully bilingual team of providers who can evaluate patients using the (S)TAAR model without the use of interpreting services.

\section{Limitations}

Outcomes should be interpreted in the context of key limitations. First, it is important to note that the 12 months of data collection in this study happened to correspond with the hiring of a nurse practitioner and psychologist who were involved in (S)TAAR's core assessment team. While this has obvious implications for improving the (S)TAAR model's capacity to take on new patients and get them seen sooner, the degree to how much this improved patient throughput and decreased wait-time is difficult to tease out without a different research design. Second, the study was conducted retrospectively to evaluate a clinical program after implementation. Third, results reflect outcomes in a unique catchment area in Central Texas with a large Spanish-speaking population and may not be generalizable to other settings and populations where there is not the clinic infrastructure to accommodate for Spanish-language evaluations and interpretation services.

\section{Conclusions}

This study evaluated the (Sin Exclusión) Transdisciplinary Autism Assessment and Resources ([S]TAAR) model on improving efficiency and equity in access to high-quality comprehensive autism evaluations for young children. Incorporating existing community-based behavioral screening and developmental testing, as well as synchronous evaluation procedures within a transdisciplinary team, improved efficiency and equity in access to high-quality comprehensive autism evaluations for young children. Reductions in waitlist and overall time to diagnosis were documented over the study period. Improvements in access to care were equitable 
across patient race, ethnicity, language, and insurance type. Availability of developmental screening/testing for pre-visit care coordination was associated with a more efficient diagnostic process.

Author Contributions All authors contributed to study conceptualization, material preparation and data collection, prepararation, writing and editing of the manuscript, and approved the final version of the manuscript. Briana Brukilacchio performed the statistical analysis.

\section{Declarations}

Conflict of interest The authors declare they have no conflicts of interest related to this work.

Ethical Approval The study was approved by the University of Texas at Austin IRB (\#2020-03-0040) as a research study involving human participants.

Informed Consent A waiver of informed consent was obtained for the retrospective chart review portion of the study and informed consent was obtained for the satisfaction portion of the study.

\section{References}

Austin, J., Manning-Courtney, P., Johnson, M., Weber, R., Johnson, H., Murray, D., Ratliff-Schaub, K., Tadlock, A. M., \& Murray, M. (2016). Improving access to care at autism treatment centers: A systems analysis approach. Pediatrics, 137, S149-S157. https:// doi.org/10.1542/peds.2015-2851M

Baio, J., Wiggins, L., Christensen, D. L., Maenner, M. J., Daniels, J., Warren, Z., Kurzius-Spencer, M., Zahorodny, W., Rosenberg, C. R., White, T., Durkin, M. S., Imm, P., Nikolaou, L., YearginAllsopp, M., Lee, L., Harrington, R., Lopez, M., Fitzgerald, R. T., Hewitt, A., ... Dowling, N. F. (2018). Prevalence and characteristics of autism spectrum disorder among children aged 8 years-autism and developmental disabilities monitoring network, 11 sites, United States, 2014. MMWR Surveillance Summaries, 67, 1-23. https://doi.org/10.15585/mmwr.ss6706a1

Bayley, N. (2005). Bayley Scales of Infant and Toddler Development (3rd ed.). Pearson. Retrieved June 14, 2021 from https://www. pearsonassessments.com/store/usassessments/en/Store/Profession al-Assessments/Behavior/Adaptive/Bayley-Scales-of-Infant-andToddler-Development-\%7C-Third-Edition/p/100000123.html

Bishop-Fitzpatrick, L., \& Kind, A. J. H. (2017). A scoping review of health disparities in autism spectrum disorder. Journal of Autism and Developmental Disorders, 47, 3380-3391. https://doi.org/10. 1007/s10803-017-3251-9

Bridgemohan, C., Bauer, N. S., Nielsen, B. A., DeBattista, A., RuchRoss, H., Paul, L., \& Roizen, N. (2018). A workforce survey on developmental-behavioral pediatrics. Pediatrics, 141, e20172164. https://doi.org/10.1542/peds.2017-2164

Crossman, M., Lindly, O., Chan, J., Eaves, M., Kuhlthau, K. A., Parker, R. A., Coury, D. L., Zand, D. H., Nowinski, L. A., Smith, K., Tomkinson, M., \& Murray, D. S. (2020). Families' experiences with family navigation services in the autism treatment network. Pediatrics, 145, S60-S71. https://doi.org/10.1542/peds/ 2019-1895I

Dababnah, S., Shaia, W. E., Campion, K., \& Nichols, H. M. (2018). "We had to keep pushing": Caregivers' perspectives on autism screening and referral practices of Black children in primary care. Intellectual and Developmental Disabilities, 56, 321-336. https:// doi.org/10.1352/1934-9556-56.5.321

Dreyer, B. (2016). AAP statement on US Preventive Services Task Force final recommendation statement on autism screening. Retrieved June 14, 2021 from https://www.org/en-us/aboutthe-aap/aap-press-room/Pages/AAP-Statement-on-US-Preve ntive-Services-Task-Force-Final-Recommendation-Statementon-Autism-Screening

Durkin, M. S., Maenner, M. J., Meaney, J., Levy, S. E., DiGuiseppi, C., Nicholas, J. S., Kirby, R. S., Pinto-Martin, A., \& Shieve, L. A. (2010). Socioeconomic inequity in the prevalence of autism spectrum disorder: Evidence from a U.S. cross-sectional study. PLoS One, 5, 11551. https://doi.org/10.1371/journal.pone.0011551

Georgetown University Health Policy Institute, Center for Children and Families. (2021). Getting back on track: A detailed look at health coverage trends for Latino children appendix. Retrieved January 8, 2022 from https://ccf.georgetown.edu/2021/06/ 08/getting-back-on-track-a-detailed-look-at-health-coveragetrends-for-latinochildren-appendix/

Gerdts, J. A., Mancini, J., Fox, E., Rhoads, C., Ward, T., Easley, E., \& Bernier, R. A. (2018). Interdisciplinary team evaluation: An effective method for the diagnostic assessment of autism spectrum disorder. Journal of Developmental and Behavioral Pediatrics, 39, 271-281. https://doi.org/10.1097/DBP.00000 00000000549

Gordon-Lipkin, E., Foster, J., \& Peacock, G. (2016). Whittling down the wait time: Exploring models to minimize the delay from initial concern to diagnosis and treatment of autism spectrum disorder. Pediatric Clinics of North America, 63, 851-859. https://doi.org/ 10.1016/j.pcl.2016.06.007

Harris, P. A., Taylor, R., Thielke, R., Payne, J., Gonzalez, N., \& Conde, J. G. (2009). Research electronic data capture (REDCap)-a metadata-driven methodology and workflow process for providing translational research informatics support. Journal of Biomedical Informatics, 42, 377-381. https://doi.org/10.1016/j.jbi. 2008.08.010

Hine, J. F., Herrington, C. G., Rothman, A. M., Mace, R. L., Patterson, B. L., Carlson, K. L., \& Warren, Z. E. (2018). Embedding autism spectrum disorder diagnosis within the medical home: Decreasing wait times through streamlined assessment. Journal of Autism and Developmental Disorders, 48, 2846-2853. https://doi.org/10. 1007/s10803-018-3548-3

Holley, M. I., Ravenscroft, S., Brukilacchio, B., Engelman, M., \& Fikki, A. (2020). Dell Children's (S)TAAR model of early autism assessment. In M. McClain, J. Shahidullah \& K. Mezher (Eds.), Interprofessional care coordination for pediatric autism spectrum disorder (pp. 65-84). https://doi.org/10.1007/ 978-3-030-46295-6_6

Jimenez, M. E., Alcaraz, E. M., Williams, J., \& Strom, B. L. (2017). Access to developmental pediatrics evaluations for at-risk children. Journal of Developmental and Behavioral Pediatrics, 38, 228-232. https://doi.org/10.1097/DBP.0000000000000427

Koushik, N. S., Bacon, B., \& Stancin, T. (2015). An interprofessional care model for evaluating autism spectrum disorders (ASDs) among low-income children. Clinical Practice in Pediatric Psychology, 3, 108-119. https://doi.org/10.1037/cpp0000093

Lappé, M., Lau, L., Dudovitz, R. N., Nelson, B. B., Karp, E. A., \& Kuo, A. A. (2018). The diagnostic odyssey of autism spectrum disorder. Pediatrics, 141, S272-S279. https://doi.org/10.1542/ peds.2016-4300c

Lipkin, P. H., \& Macias, M. M. (2020). Promoting optimal development: Identifying infants and young children with developmental disorders through developmental surveillance and screening. Pediatrics, 145, e20193449. https://doi.org/10.1542/peds.2019-3449 
Newborg, J. (2009). Battelle developmental inventory-second edition. Itasca: Riverside.

Parker, M. L., Diamond, R. M., \& Del Guericio, A. D. (2019). Care coordination of autism spectrum disorder: A solution-focused approach. Issues in Mental Health Nursing, 41, 138-145. https:// doi.org/10.1080/01612840.2019.1624899

Phelps, R., \& Coker, T. (2019). First, do no harm: Improving access in autism diagnostic assessments for children without exacerbating inequities in care. Journal of Developmental and Behavioral Pediatrics, 40, 217-218. https://doi.org/10.1097/DBP.00000 00000000663

Radecki, L., Sand-Loud, N., O'Connor, K. G., Sharp, S., \& Olson, L. M. (2011). Trends in the use of standardized tools for developmental screening in early childhood: 2002-2009. Pediatrics, 128, 14-19. https://doi.org/10.1542/peds.2010-2180

Robins, D. L., Fein, D., \& Barton, M. (2009). Modified checklist for autism in toddlers, revised with follow-up (M-CHAT-R/F). 2009. Retrieved June 14, 2021 from https://mchatscreen.com/wp-conte nt/uploads/2015/09/M-CHAT-R_F_Rev_Aug2018.pdf

Schopler, E., Bourgondien, M. E. V., Wellman, G. J., \& Lowe, S. r. (2010). Childhood Autism Rating Scale-Second Edition (CARS2). Retrieved June 14, 2021 from https://www.pearsonclinical.co. uk/Psychology/ChildMentalHealth/ChildAutisticSpectrumDiso rders/ChildhoodAutismRatingScale-SecondEdition(CARS2)/ ChildhoodAutismRatingScale-SecondEdition(CARS2).aspx

Shahidullah, J. D., Azad, G. F., Mezher, K., McClain, M. B., \& McIntrye, L. (2018). Linking the medical and educational home to support children with autism spectrum disorder: Practice recommendations. Clinical Pediatrics, 57, 1496-1505. https://doi.org/ 10.1177/0009922818774344

Shahidullah, J. D., McClain, M. B., Azad, G., Mezher, K. R., \& McIntyre, L. (2020). Coordinating autism care across schools and medical settings: Considerations for school psychologists. Intervention in School and Clinic, 56, 107-114. https://doi.org/10.1177/10534 51220914891

Shanafelt, T. D., Boone, S., Tan, L., Dyrbye, L. N., Sotile, W., Satele, D., West, C. P., Sloan, J., \& Oreskovich, M. R. (2012). Burnout and satisfaction with work-life balance among US physicians relative to the general US population. Archives of Internal Medicine, 172, 1377-1385. https://doi.org/10.1001/archinternmed.2012. 3199

Shanafelt, T., Goh, J., \& Sinsky, C. (2017). The business case for investing in physician well-being. JAMA Internal Medicine, 177, 1826-1832. https://doi.org/10.1001/jamainternmed.2017.4340

Sheldrick, R. C., Maye, M. P., \& Carter, A. S. (2017). Age at first identification of autism spectrum disorder: An analysis of two US surveys. Journal of the American Academy of Child and Adolescent Psychiatry, 56, 313-320. https://doi.org/10.1016/j.jaac. 2017.01.012

Squires, J., \& Bricker, D. (2019). Ages \&amp; Stages Questionnaires ${ }^{\circledR}$, Third Edition (ASQ®-3): A parent-completed child monitoring system. Paul H. Brookes Publishing Co., Inc.

Thomas, K. C., Parish, S. L., Rose, R. A., \& Kilany, R. (2012). Access to care for children with autism in the context of state Medicaid reimbursement. Maternal Child Health Journal, 16, 1638-1644. https://doi.org/10.1007/s10995-011-0862-1

Voress, J. K., Maddox, T., \& Hammill, D. D. (2012). Developmental assessment of young children, second edition. Pearson. Retrieved June 14, 2021 from https://www.pearsonassessments.com/store/ usassessments/en/Store/Professional-Assessments/Developmen tal-Early-Childhood/Developmental-Assessment-of-Young-Child ren-\%7C-Second-Edition/p/100000730.html

Vyt, A. (2008). Interprofessional and transdisciplinary teamwork in health care. Diabetes/metabolism Research and Reviews, 24, S106-S109. https://doi.org/10.1002/dmrr.835

West, C. P., Dyrbye, L. N., \& Shanafelt, T. D. (2018). Physician burnout: Contributors, consequences and solutions. Journal of Internal Medicine, 283, 516-529. https://doi.org/10.1111/joim.12752

Williams-Arya, P., Anixt, J., Kuan, L., Johnson, H., Kent, B., Bing, N., Ehrhardt, J., \& Manning-Courtney, P. (2019). Improving access to diagnostic assessments for autism spectrum disorder using an arena model. Journal of Developmental and Behavioral Pediatrics, 40, 161-169. https://doi.org/10.1097/DBP.0000000000 000648

Woodgate, R. L., Ateah, C., \& Secco, L. (2008). Living in a world of our own: The experience of parents who have a child with autism. Qualitative Health Research, 18, 1075-1083. https://doi.org/10. 1177/1049732308320112

World Health Organization. (2008). Task-shifting: global recommendations and guidelines. Retrieved June 14, 2021 from https://www. who.int/workforcealliance/knowledge/resources/taskshifting_ guidelines/en/

Zablotsky, B., Black, L. I., \& Blumberg, S. J. (2017). Estimated prevalence of children diagnosed with developmental disabilities in the United States, 2014-2016. U.S. Department of Health and Human Services, National Center for Health Statistics Data Brief, 291. Retrieved June 14, 2021 from https://pubmed.ncbi.nlm.nih.gov/ 29235982/

Zuckerman, K. E., Lindly, O. J., \& Sinche, B. K. (2015). Parental concerns, provider response, and timeliness of autism spectrum disorder diagnosis. Journal of Pediatrics, 166, 1431-1439. https:// doi.org/10.1016/j.jpeds.2015.03.007

Zwaigenbaum, L., Bauman, M. L., Stone, W. L., Yirmiya, N., Estes, A., Hansen, R. L., McPartland, J. C., Natowicz, M. R., Choueiri, R., Fein, D., Kasari, C., Pierce, K., Buie, T., Carter, A., Davis, P. A., Granpeesheh, D., Mailloux, Z., Newschaffer, C., Robins, D., ... Wetherby, A. (2015). Early identification of autism spectrum disorder: Recommendations for practice and research. Pediatrics, 136, S10-S40. https://doi.org/10.1542/peds.2014-3667C

Publisher's Note Springer Nature remains neutral with regard to jurisdictional claims in published maps and institutional affiliations. 